\title{
MMaPFlow: A Crowd-sourcing based Approach for Mapping Mass Pedestrian Flow
}

\author{
Emad Felemban ${ }^{1,3,4}$, Adil A. Sheikh ${ }^{1,2,3}$, Faisal Karim Shaikh ${ }^{1,2,3,5}$ \\ ${ }^{1}$ Simplicity Labs, ${ }^{2}$ Science and Technology Unit \\ ${ }^{3}$ Transportation and Crowd Management Center of Rearch Excellence \\ ${ }^{4}$ Dept. of Comp. Engg., Umm Al-Qura University, Saudi Arabia \\ ${ }^{5}$ Mehran University of Engineering \& Technology, Pakistan \\ \{eafelemban,aasheikh,fkshaikh\}@uqu.edu.sa
}

\begin{abstract}
Mass gatherings and mass pedestrian flows such as during holy rituals are always challenging for the management as well as for the people attending them. The holy city of Makkah hosts millions of pilgrim coming from all over the world each year during Ramadan and Hajj seasons. One of the main rituals is the tawaaf (circumambulation) of the Holy Kaaba. Knowing the average tawaaf time and flow rates of pilgrims around Holy Kaaba is beneficial in order to efficiently schedule the visit to the Sacred Mosque and avoid crowdedness. This paper discusses MMaPFlow approach for Mapping Mass Pedestrian Flow around Holy Kaaba using crowd-sourcing. The crowd-sourcing is done through pilgrim smartphone to calculate average tawaaf time and other statistics related to pilgrims movement around the Holy Kaaba.
\end{abstract}

\section{Categories and Subject Descriptors}

H.4 [Information Systems Applications]: Miscellaneous; D.2.8 [Software Engineering]: Metrics-complexity measures, performance measures

\section{General Terms}

Algorithms, Design, Experimentation, Human Factors, Measurement

\section{Keywords}

Crowd-sourcing, Crowd Flow, Tawaaf, Kaaba

\section{INTRODUCTION}

Crowd management and monitoring crowd flow is essential for the safety of people in public areas as well as in some special events such as holy gatherings. One of the biggest event of gathering is performance of Hajj in Makkah Saudi Arabia. Hajj is compulsory for all the muslims who can afford it. Hence, most muslims visit the Sacred Mosque in Makkah at least once in their lifetime during a specific period

Permission to make digital or hard copies of all or part of this work for personal or classroom use is granted without fee provided that copies are not made or distributed for profit or commercial advantage and that copies bear this notice and the full citation on the first page. To copy otherwise, to republish, to post on servers or to redistribute to lists, requires prior specific permission and/or a fee.

CSSWearable 2014, December 02-04, London, Great Britain

Copyright @ 2014 ICST 978-1-63190-039-6

DOI 10.4108/icst.mobiquitous.2014.257985 of Hajj. According to the Saudi Arabian Central Department of Statistics and Information almost 3 million people performed Hajj in 2012 [1]. Saudi government is pursuing to host even a larger number of pilgrimage through various infrastructure expansion projects including Sacred Mosque and its surrounding residential areas, highways, rails, metro lines and road networks. After the completion of the ongoing expansion project, the capacity of the Sacred Mosque will increase considerably. Other than Hajj, muslims visit the Sacred Mosque all year round for Umrah. One of the primary ritual of Hajj and Umrah is the tawaaf of Holy Kaaba. A complete tawaaf consists of walking counter-clockwise seven times around the Holy Kaaba. Tawaaf takes place in the central piazza of Sacred Mosque as well as dedicated pathways of Sacred Mosque building. When the main piazza is not crowded (Fig. 1(a)), it takes on the average 20-25 minutes to perform the seven circumambulations for a normal person. However, when the main piazza is overcrowded (Fig. 1(b)), it may take hours [1] as shown in Fig.1.

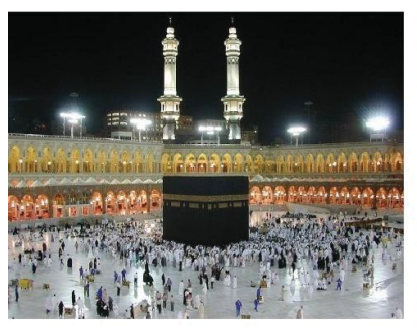

(a) Sparsely Crowded

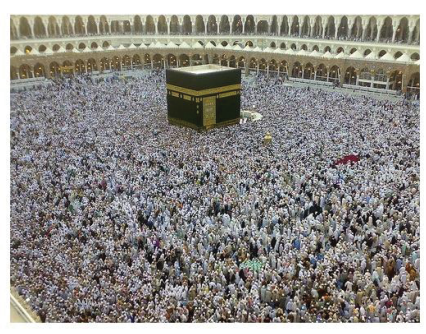

(b) Densely Crowded
Figure 1: Densely Crowded Vs. Sparsely Crowded Piazza Area around Holy Kaaba

Scheduling a pilgrim's visit to the Sacred Mosque for tawaaf as well as planning maintenance and cleaning activities of the central piazza requires statistics such as average tawaaf time, tawaaf flow rates and crowdedness at any point of the day, whole year round in the central piazza. Pilgrims can schedule their visit to the Sacred Mosque for tawaaf if they are aware of suitable timings for it. Likewise, cleaning and maintenance can be scheduled when the central piazza is least crowded so that least pilgrims are disturbed.

Collection of pilgrim movement data can be achieved through many different means. In this paper, we exploit the emerging phenomenon of crowd-sourcing using smartphones of the pilgrims. The information is collected on the server in a real- 
time manner and different statistics such as average tawaaf time, crowd density etc, will be available to the concerned people. The main challenge is to meaningfully transform the crowd-sourced data in usable form and having huge number of people involved in the crowd scenarios.

The rest of the paper is organized as follows. Section 2 discusses the related work. Section 3 explains our approach. Section 4 provides details of how we evaluate our framework. Section 5 provide insights of the results. Section 6 list the challenges faced during the deployment and collection of data and how we address them. Section 7 concludes the paper.

\section{RELATED WORK}

The data from the crowd can be gathered using many techniques [2]. They are based on image processing, Global Positioning System (GPS), pressure sensor, inertial sensor, radio signal (WiFi, Bluetooth, RFID, etc.), and crowd-sourcing.

Mori et al. [3] successfully tracked up to 3 persons by embedding pressure sensors in the floor and asking pedestrians to wear RFID tags in their shoes. They track people inside a building where they have installed their pressure sensors and RFID readers. Nazemzadeh et al. [4] presents a position tracking algorithm based on an extended Kalman filter that combines odometer and gyroscope data with passive RFID tag and visual marker detection.

Colombo et al. [5] discuss their local positioning system comprising of a wearable embedded inertial platform with wireless connectivity. They are interested in locating people inside homes as well as logging their activity using gyroscopes, magnetometers and accelerometers. Alzantot et al. [6] used inertial sensors in smartphones to track people by counting steps and step sizes. Radu et al. [7] and Zhang et al. [8] also use smartphone inertial sensors for indoor tracking.

Falcone et al. [9] used bi-static passive WiFi radars to track vehicles. Broetje et al. [10] extended the work of Falcone et al. by using WiFi based multistatic passive radars to track the people.

Rahimi et al. [11] use a 3 camera network to detect and track people using support vector machine classifiers. They use color and histograms of oriented gradients (HOG) to model the humans. Zhang et al. [12] use 2 camera network to do the same. Rao et al. [13] proposed a block-based dense optical flow to obtain crowd velocities. They process video footage to extract approximate crowd density and flow. Sherrah et al. [14] also process video to extract head and body of pedestrians using HOG detectors. Their experiments show that the use of head and body detections along with multiple hypothesis tracking improves track-by-detect methods.

Adiaviakoye et al. [15] successfully tracked 300 students in the school's lobby using laser range scanners. In $[16,17]$ laser range scanners are used for the localization of people.

Several studies have been carried out that use subscriber location data from cell phone towers to locate a mobile phone. Calabrese et al. [18] used subscriber location data to monitor urban movement in real-time. Other researchers in $[19$,
20, 21] have also carried out similar studies. Mitchell et al. [22] calculated tawaaf statistics using RFID tags augmented with mobile phone data. Bilgic et al. [23] used GPSenabled phones to track persons.

The first of these techniques requires embedding sensor below the floor. This is not possible in our case. The second techniques of using gyro, accelerometer and magnetometer focusing on indoor navigation. This technique is similar to ours, however we use GPS only focusing on outdoor pilgirm movement only. The third techniques uses WiFi radars. The fourth technique uses cameras. The fifth techniques uses laser range finders. None of these can be implemented in the Sacred Mosque without infrastructural changes. Finally, the last technique uses cell phone tower data which is unavailable to us.

\section{MMAPFLOW: A CROWD-SOURCING AP- PROACH}

The ritual of Tawaaf is performed counter-clockwise around the Holy Kaaba in the Sacred Mosque's central piazza as well on the dedicated bridges and on the roof of the mosque. In the rest of the paper we concentrate over the central piazza but the proposes approach can be easily adopted for the specialized bridges as well as for the roof of the mosque.
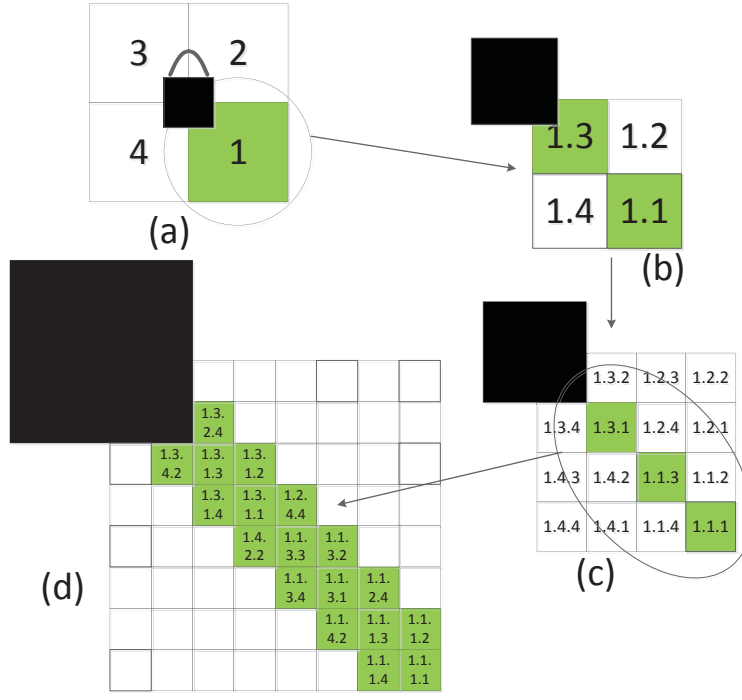

Figure 2: Virtual Quadrants of Main Piazza Area

Tawaaf starts from south-east corner of the Holy Kaaba where the black stone is placed. Each round starts and ends at the same corner. In order to monitor the pedestrian movement throughout the Tawaaf it is important to find when a person comes on the start position. We divide the piazza into four quadrants as shown in Fig.2(a). We further divide each quadrant into to four sub-quadrants as shown in Fig.2(b). For more granularity the sub-quadrants can be further divide two more times as shown in Fig.2(d). The green squares as shown in Fig. 2(d) represent the approximate position of tawaaf start. When the pilgrim reaches the central piazza and starts performing tawaaf, the smartphone application starts logging pilgrim location and sends it to the 
server in real-time using $3 \mathrm{G}$ or $4 \mathrm{G}$ connectivity. In case if Internet connectivity is not available the application stores the data locally and as soon as the connectivity is available the whole data is sent to our server. As the pilgrim crosses the green area of Fig.2(d), our application marks start of tawaaf and subsequently the end of a round.

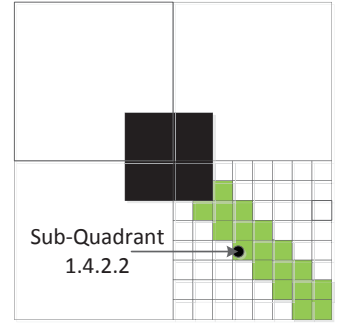

(a)

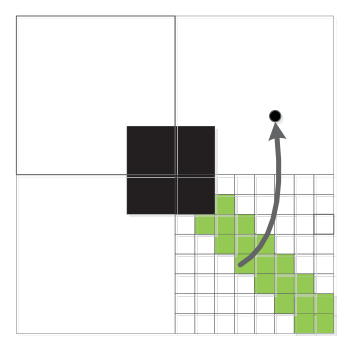

(c)

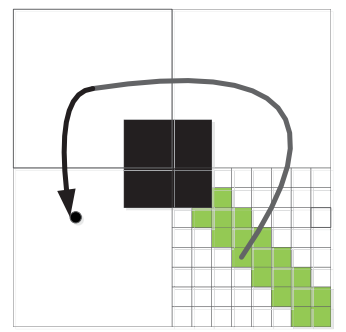

(e)

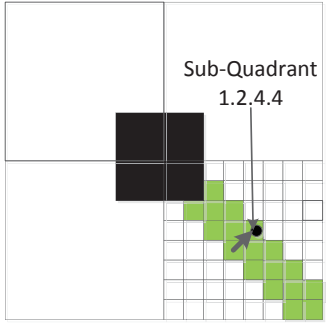

(b)

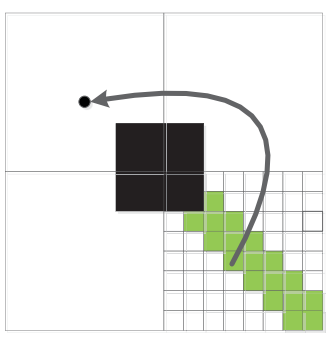

(d)

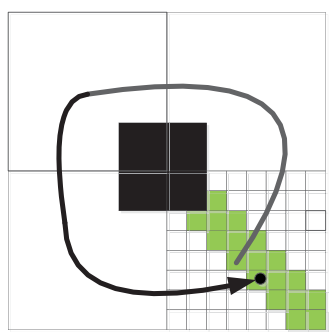

(f)
Figure 3: Movement of Pilgrim in Main Piazza Area

Let us consider when the server receives a location within a sub-quadrant 1.4.2.2 (depicted in Fig.3(a)). Our server will mark the start of tawaaf for a pilgrim. As the pilgrim moves further, we receive another location from subquadrant 1.2.4.4 (Fig.3(b)) to map its movement in the piazza. In this way, we keep receiving locations till the pilgrim reaches quadrant 2 . The history of pilgrim movement is logged. Then, the pilgrim moves to quadrant 3, 4 and finally back to 1 . The complete movement is depicted in Fig.3(a-f)). In quadrant 1, when the pilgrim reaches to any of the green sub-quadrant, one circumambulation or tawaaf cycle is complete.

\section{EVALUATION}

This section explains how we evaluated our approach for mapping the mass pedestrian movement.

\subsection{Application}

Crowd-sourced Android and iPhone applications have spawned the participation of crowd for data collection for various studies which were expensive in the past. Additionally, the use of Assisted GPS (A-GPS) has made location-aware applications possible. In order to collect tawaaf data, an Android application has been developed with some incentive for the pilgrim. The pilgrims run the application on their smartphones while performing the tawaaf. The application uses A-GPS to sample the location of the pilgrim every 30 seconds. Smartphone's IMEI, time, date and sampled location is transmitted to the server as well as logged locally in smartphone's memory.

The smartphone application is called as My Tawaaf (Fig.4) and is available via Google Play Store [24].
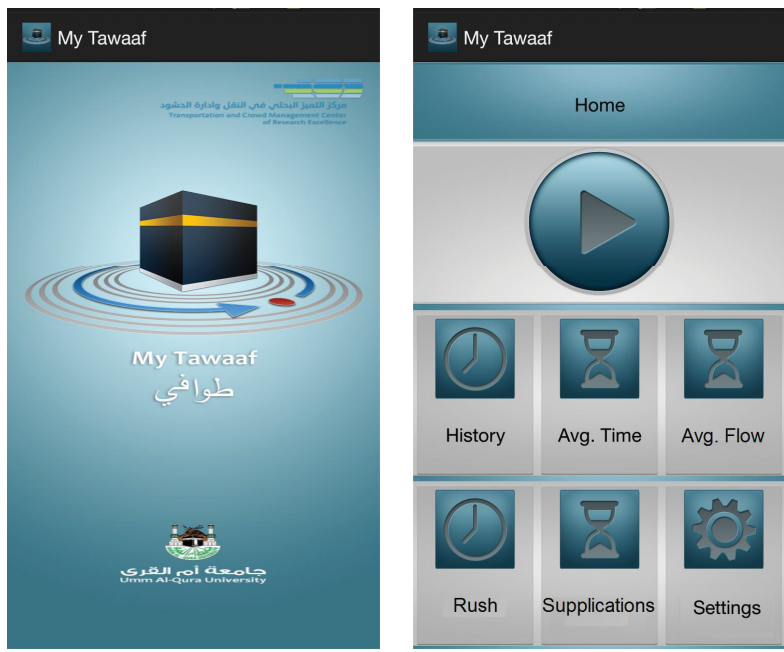

Figure 4: Splash and Main Screen of My Tawaaf

Upon startup the application checks if the GPS is enabled or not. If it is not enabled, the pilgrim is prompted to enable it. Once enabled, the application waits for the pilgrim to reach the tawaaf start area. There onwards, the application samples the required parameters and send them to the server. When the pilgrim completes one tawaaf cycle or circumambulation, the current cycle count is displayed on the screen. The pilgrim can view his/her previous tawaaf history by clicking the history button.

\subsection{Deployment}

In addition to making the application available on Internet, we distributed My Tawaaf Android application to 30 volunteer students for collecting data. We also provided students with dedicated GPS data loggers to compare the accuracy of the samples collected by the application using smartphone. The students took turns in performing tawaaf so that we have data for 24 hours a day for seven days. Hence, we collected data 345 tawafs i.e. 2415 circumambulations. Each smartphone was used two hours to collect data before it was brought back for recharging. At this time, we also copied the samples saved in the phone's memory. Augmenting the missing samples into database improves the accuracy of statistics.

\subsection{Metrics}

The following metrics were calculated to evaluate our approach. 
Average Tawaaf Cycle Time As our server logs location data along with time, finding the time difference between start of tawaaf cycle and end of tawaaf cycle gives us tawaaf time. Our system takes average of tawaaf times calculated for location data coming from all smartphones being used at that time. This gives us average tawaaf time. This metric is sent back to the smartphone so that it can be displayed in the application.

Average Tawaaf Flow Rate The server application calculates pilgrims flow rates by dividing distance covered by the pilgrim by the time it took him or her to complete one tawaaf cycle. This metric is also sent back to the smartphone so that it can be displayed in the application.

Delivery Ratio The number of location samples received by our server application may be less than the number of samples collected by the smartphone application. Hence, we are interested in the delivery ratio, that is the number of samples received over number of samples collected. My Tawaaf saves all collected locations in smartphone's internal memory. At the end of the experiment, when the student returns the smartphone, we manually upload all the collected samples to the server. With this data, the server application calculates delivery ratio by dividing number of received location samples over total samples collected by the phone. This metric has been calculated for evaluation of our smartphone and server application.

\section{RESULTS}

This section discusses the results of our evaluation.

\subsection{A-GPS Accuracy}

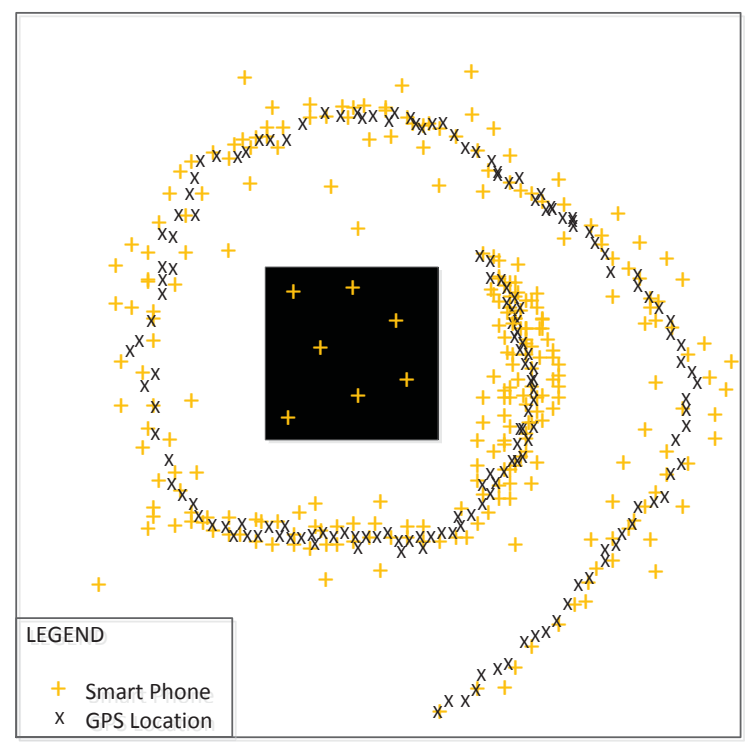

Figure 5: Comparison of Sampling Accuracy - Specialized GPS Device Vs. Smartphone A-GPS

We compared the location data gathered through our smartphone applications with data collected through specialized
GPS devices as used by Koshak et al. [25]. The specialized GPS devices provide much more accurate location data than A-GPS based smartphones. Using crowd-sourced data from A-GPS based smartphones instead of specialized GPS devices is a trade off that reduces equipment costs but increases inexpensive crowd participation. Extensive studies have already been carried out to quantify the error of AGPS based smartphones [26, 27]. Our scenario is special from many aspects thus encouraging us to have an experiment to measure the accuracy of a smartphones compared to specialized GPS receivers. Fig. 5 shows the accumulated data from one circumambulation. The crosses represent location detection using specialized GPS receivers. The pluses represent the location data collected by our smartphone application on the same circumambulation. It can be seen that the error of smartphones is greater than specialized GPS receiver's error.

\subsection{Average Tawaaf Cycle Time}

Fig.6 is one of the statistics that is available for display on our website as well as on My Tawaaf Android app. Results show that average tawaaf times are higher right before each prayer and also in evening. This result confirms the observation of an average pilgrim. A large number pilgrim visit the Sacred Mosque only for the prescribed times i.e. approximately at $5 \mathrm{am}, 12: 30 \mathrm{pm}, 4 \mathrm{pm}, 7: 00 \mathrm{pm}$ and 8:30 pm. They hasten to perform tawaaf before the prayer. As soon as prayer finishes, a lot of pilgrim leave the central piazza. This is why average tawaaf times are higher right before the prescribed prayer times.

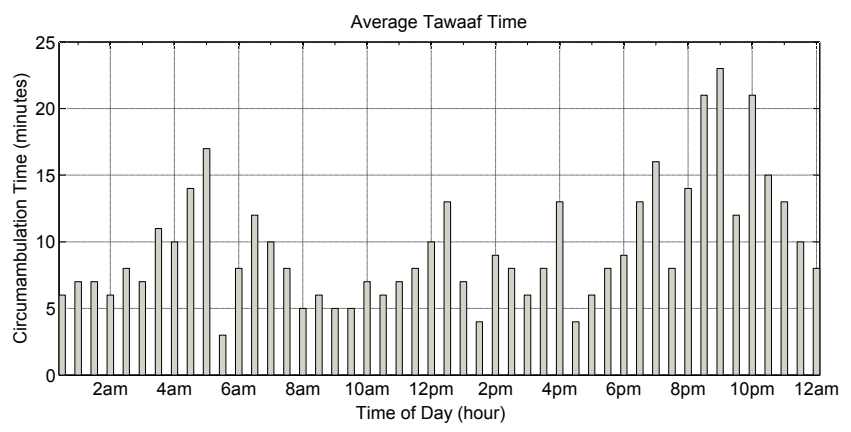

Figure 6: Average Tawaaf Cycle Time

\subsection{Average Tawaaf Flow Rate}

Fig. 7 shows the average flow rate of pilgrim performing tawaaf around the Holy Kaaba. Flows increase when the central piazza is less crowded and decreases when it is more crowded. As mentioned before, the number of pilgrim increases right before prayer times. Hence, the flow rates are lower right before prayer time and higher right after prayer time because a lot of pilgrims leave the central piazza after prayer.

\subsection{Delivery Ratio}

The server application calculates the ratio of number of location samples received live verses the number of samples actually collected. Fig. 8 shows us the delivery ratio. If Internet connectivity is unavailable, My Tawaaf is unable to upload collected location samples in real-time. The application 


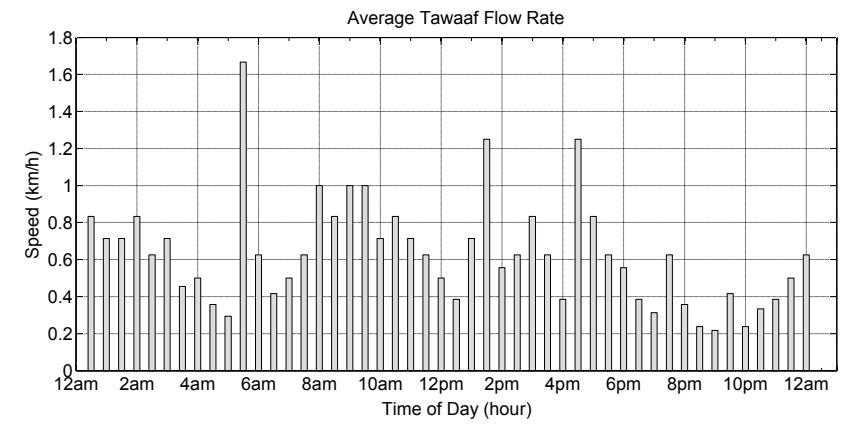

Figure 7: Average Tawaaf Flow Rate

saves un-sent samples in the smartphone's internal memory. As soon as Internet connectivity is available, the application connects with our server application and uploads the stale location samples. Fig. 8 shows a drop in delivery ratio at times when the smartphone application was unable to get Internet connectivity from mobile Internet service provider.

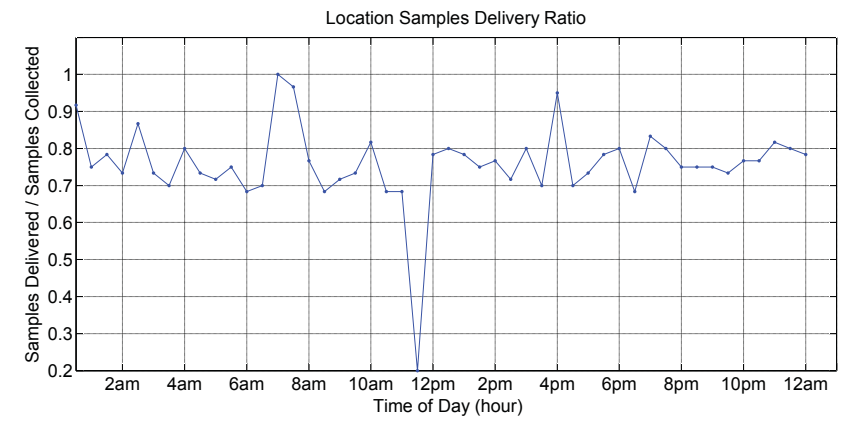

Figure 8: Delivery Ratio

\section{CHALLENGES}

Various expected and unexpected challenges were faced during implementation, data collection and calculations of tawaaf data. These are briefly explained here.

\subsection{Incentive for the Crowd}

One of the major challenges is to make sure that pilgrims share their location data with us. Without information from users, our server would not receive enough data for calculating tawaaf times for the entire day. As most crowd-sourced applications, we also faced this challenge. Researchers [28] have suggested providing incentive for the users in order to create a win-win strategy. We used the following incentives schemes for the pilgrims.

Tawaaf Cycle Count A complete tawaaf consists of seven circumambulations around the Holy Kaaba. Pilgrims prefer reciting supplications during tawaaf. Hence, they have to make little effort to remember their cycle count. Most pilgrims use special beaded strings or electronic tally counters for this purpose. As an incen- tive, the application displays the current cycle count on the smartphone screen.

History The smartphone application keeps history of previous tawaafs of the pilgrim. This also serves as an incentive for those pilgrims who want to know their personal visit statistics.

Guidance and Information As mentioned before, during tawaaf pilgrims prefer reciting supplications. Our smartphone application contains a large number of recommended supplications that pilgrims can recite during tawaaf. Guidance towards points-of-interest such as location of facilities, gates, exits, and other important landmarks are also provided. Emergency phone numbers and contact details of important service providers are also available.

Crowdedness Level Pedestrian flow rates are directly proportional to crowdedness level [29]. Based on average tawaaf times and flow rates, the application provides a crowdedness index to the pilgrims. This incentive helps pilgrims efficiently planning their visit to the Sacred Mosque.

\subsection{Optimal Data Size}

To provide live tawaaf statistics all year around, our server needs constant data collection. However, there are large spans of times when none of the pilgrims performing tawaaf in the Sacred Mosque is using our smartphone application. This data scarcity causes our system not to provide tawaaf statistics at these times. At these times, our server provides either interpolated tawaaf statistics or stale results both of which are not live. Introducing incentives has improved this challenge. Another mitigation methodology is to send probe pilgrims similar to the concept of sending probe vehicles to collect data when not enough crowd-sourced data collection vehicles are available [30].

Some pilgrims turn off their mobile's data connection to conserve battery and save additional costs. In this case, our server does not receive live location data from the smartphones. This challenge cannot be solved until more incentive is provided to the pilgrims, however, it can be alleviated. Our smartphone application keeps saving pilgrim's location on the smartphone in a local database. Whenever Internet connectivity is available, the database synchronizes its samples with our server. In this way, we receive pilgrim's stale location data. This data is still of use to us in order to provide average tawaaf time, flow rate trends and histories.

As compared to lack of data, too much data is also a problem which chokes our data collection server. Although this challenge has not been faced till now, we anticipate it during the Hajj season. Our mitigation plan is implement collaboration between smartphones applications running in main piazza. If the number of live smartphones application increase a certain threshold, we will schedule data coming from smartphones. Each smartphone can be given a time slot in which it will transmit the location data it has accumulated. Another strategy is to remotely disable data collections from a set of smartphones. 


\section{CONCLUSIONS AND FUTURE WORK}

In this paper, we discussed the architecture and challenges we faced during development of our approach for mapping the pedestrian movement during tawaaf. We focused on gathering data from crowd-sourced smartphone applications. In future, we wish to quantify the robustness, efficiency, scalability and reliability of crowd-sourced smartphone application based location detection and compare it with other methodologies.

\section{ACKNOWLEDGMENTS}

This work is sponsored by the Transportation and Crowd Management Center of Research Excellence (TCMCORE) at the University of Umm Al-Qura, Makkah. We would like to thank Abdulhannan Rashid, Abdullah Hawsawi, Mohammad Sabir and other TCMCORE employees for their valuable support.

\section{REFERENCES}

[1] X. Zamani. Central department of statistics and information, the number of pilgrims for the years from (1995g.) to (2010g.), 2010. http://www.cdsi.gov.sa, Date Accessed: 08-17-2014.

[2] Beibei Zhan, Dorothy N. Monekosso, Paolo Remagnino, Sergio A. Velastin, and Li-Qun Xu. Crowd analysis: A survey. Mach. Vision Appl., 19(5-6):345-357, September 2008.

[3] T. Mori, Y. Suemasu, H. Noguchi, and Tomomasa Sato. Multiple people tracking by integrating distributed floor pressure sensors and rfid system. In Systems, Man and Cybernetics, 2004 IEEE International Conference on, volume 6, pages 5271-5278 vol.6, Oct 2004.

[4] P. Nazemzadeh, D. Fontanelli, D. Macii, and L. Palopoli. Indoor positioning of wheeled devices for ambient assisted living: A case study. In Instrumentation and Measurement Technology Conference (I2MTC) Proceedings, 2014 IEEE International, pages 1421-1426, May 2014.

[5] A Colombo, D. Fontanelli, D. Macii, and L. Palopoli. A wearable embedded inertial platform with wireless connectivity for indoor position tracking. In Instrumentation and Measurement Technology Conference (I2MTC), 2011 IEEE, pages 1-6, May 2011.

[6] M. Alzantot and M. Youssef. Uptime: Ubiquitous pedestrian tracking using mobile phones. In Wireless Communications and Networking Conference (WCNC), 2012 IEEE, pages 3204-3209, April 2012.

[7] V. Radu and M.K. Marina. Himloc: Indoor smartphone localization via activity aware pedestrian dead reckoning with selective crowdsourced wifi fingerprinting. In Indoor Positioning and Indoor Navigation (IPIN), 2013 International Conference on, pages $1-10$, Oct 2013.

[8] Lei Zhang, Jiangchuan Liu, Hongbo Jiang, and Yong Guan. Senstrack: Energy-efficient location tracking with smartphone sensors. Sensors Journal, IEEE, 13(10):3775-3784, Oct 2013.

[9] P. Falcone, F. Colone, A Macera, and P. Lombardo. Localization and tracking of moving targets with wifi-based passive radar. In Radar Conference
(RADAR), 2012 IEEE, pages 0705-0709, May 2012.

[10] M. Broetje. Person tracking for wifi based multistatic passive radar. In Information Fusion (FUSION), 2013 16th International Conference on, pages 280-287, July 2013.

[11] S. Rahimi, A Aghagolzadeh, and H. Seyedarabi. Detection and tracking of occluded humans in three-camera network. In Robotics and Mechatronics (ICRoM), 2013 First RSI/ISM International Conference on, pages 32-37, Feb 2013.

[12] Zhognchuan Zhang and F. Cohen. 3d pedestrian tracking based on overhead cameras. In Distributed Smart Cameras (ICDSC), 2013 Seventh International Conference on, pages 1-6, Oct 2013.

[13] AS. Rao, J. Gubbi, S. Marusic, P. Stanley, and M. Palaniswami. Crowd density estimation based on optical flow and hierarchical clustering. In Advances in Computing, Communications and Informatics (ICACCI), 2013 International Conference on, pages 494-499, Aug 2013.

[14] J. Sherrah, B. Ristic, and D. Kamenetsky. A pedestrian multiple hypothesis tracker fusing head and body detections. In Digital Image Computing: Techniques and Applications (DICTA), 2013 International Conference on, pages 1-8, Nov 2013.

[15] L. Adiaviakoye, P. Patrick, B. Marc, and J.-M. Auberlet. Tracking of multiple people in crowds using laser range scanners. In Intelligent Sensors, Sensor Networks and Information Processing (ISSNIP), 2014 IEEE Ninth International Conference on, pages 1-6, April 2014.

[16] A Nuchter, K. Lingemann, J. Hertzberg, and H. Surmann. Accurate object localization in 3d laser range scans. In Advanced Robotics, 2005. ICAR '05. Proceedings., 12th International Conference on, pages 665-672, July 2005.

[17] Mariusz Olszewski, Barbara Siemiatkowska, Rafal Chojecki, Piotr Marcinkiewicz, Piotr Trojanek, and Marek Majchrowski. Mobile robot localization using laser range scanner and omnicamera. In Teresa ZieliÅĎska and Cezary ZieliÅĎski, editors, Romansy 16, volume 487 of CISM Courses and Lectures, pages 447-454. Springer Vienna, 2006.

[18] F. Calabrese, M. Colonna, P. Lovisolo, D. Parata, and C. Ratti. Real-time urban monitoring using cell phones: A case study in rome. Intelligent Transportation Systems, IEEE Transactions on, 12(1):141-151, March 2011.

[19] Y.B. Yim and Cayford. Investigation of Vehicles as Probes Using Global Positioning System and Cellular Phone Tracking: Field Operational Test. California PATH working paper. California PATH Program, Institute of Transportation Studies, University of California at Berkeley, 2001.

[20] J. White, J. Quick, and P. Philippou. The use of mobile phone location data for traffic information. In Road Transport Information and Control, 2004. RTIC 2004. 12th IEE International Conference on, pages 321-325, April 2004.

[21] R. Herring, A. Hofleitner, S. Amin, T. Abou Nasr, A. Abdel Khalek, P. Abbeel, and A. Bayen. Using mobile phones to forecast arterial traffic through 
statistical learning. In Proceedings of the 89th Annual Meeting of the Transportation Research Board, Washington D.C., 2010.

[22] R.O. Mitchell, H. Rashid, F. Dawood, and A AlKhalidi. Hajj crowd management and navigation system: People tracking and location based services via integrated mobile and rfid systems. In Computer Applications Technology (ICCAT), 2013 International Conference on, pages 1-7, Jan 2013.

[23] H.T. Bilgic and AZ. Alkar. A secure tracking system for gps-enabled mobile phones. In Information Technology and Multimedia (ICIM), 2011 International Conference on, pages 1-5, Nov 2011.

[24] TCMCORE. My tawaaf, 2014. http://play.google.com, Date Accessed: 08-17-2014.

[25] Nabeel Koshak and Abdullah Fouda. Analyzing pedestrian movement in mataf using gps and gis to support space redesign. In Proceedings of the 9th international conference on design and decision support systems in architecture and urban planning, 2008.

[26] Klaus ten Hagen Marko Modsching, Ronny Kramer.
Field trial on gps accuracy in a medium size city: The influence of built-up. In Proceedings of the 3rd workshop on positioning, navigation and communicaiton, 2006.

[27] Soyoung Hwang and Donghui Yu. Gps localization improvement of smartphones using built-in sensors. International Journal of Smart Home, 6(3), 2012.

[28] R. Sen. Rasteyrishtey: A social incentive system to crowdsource road traffic information in developing regions. In Mobile Computing and Ubiquitous Networking (ICMU), 2014 Seventh International Conference on, pages 171-176, Jan 2014.

[29] Junping Zhang, Ben Tan, Fei Sha, and Li He. Predicting pedestrian counts in crowded scenes with rich and high-dimensional features. Trans. Intell. Transport. Sys., 12(4):1037-1046, December 2011.

[30] Wang Li, Wang Chuanjiu, Shen Xiaorong, and Fan Yuezu. Probe vehicle sampling for real-time traffic data collection. In Intelligent Transportation Systems, 2005. Proceedings. 2005 IEEE, pages 222-224, Sept 2005. 